\title{
Configuration of a Monopulse Antenna Assembly for Small Diameter Flight Vehicle Applications
}

\author{
Jaesik Kim*
}

\begin{abstract}
In this letter, configuration of a monopulse direction-finding (DF) antenna assembly is presented which can be applied for small diameter flight vehicle applications. The assembly consists of five antennas which consist of four radome mounted antennas and a slot fed cavity antenna. All antennas are located inside of a tangent ogive radome of $1.52 \lambda_{c}$ inner radius where $\lambda_{c}$ corresponds to the wavelength of the center frequency. To verify the DF performance of the antenna assembly, sum $(\Sigma)$ and delta $(\Delta)$-patterns are measured and its $\Delta / \Sigma$ monopulse curves are presented.
\end{abstract}

Key Words: Compact Antennas, Direction-Finding Antennas, Monopulse Antennas, Waveguide Antennas.

\section{INTRODUCTION}

To achieve portability, cost effectiveness, and lightness of direction-finding (DF) flight vehicle applications, compact DF antennas are recommended. In this letter, new configuration of a monopulse DF antenna assembly is presented which can be fitted in a small radius of $1.52 \lambda_{c}$, where $\lambda_{c}$ corresponds to the wavelength of the center frequency. Due to the limited space of the platform, representative monopulse antenna types, such as Cassegrain reflectors with monopulse feeds, slot array antennas, horn clusters, microstrip monopulse antennas, are difficult to apply when their dimensions, configurations, and environmental robustness are considered simultaneously [1-5]. The presented assembly consists of four radome mounted antennas and a slot fed cavity antenna. To verify the validation of the proposed configuration, the antenna assembly is designed at $\mathrm{Ku}-$ band and its sum $(\Sigma)$, delta $(\Delta)$-patterns, and $\Delta / \Sigma$ monopulse curve are measured.

\section{CONFIGURATION OF AN ANTENNA ASSEMBLY}

Fig. 1(a) illustrates the configuration of the proposed antenna assembly. The assembly consists of a radome, a slot fed cavity antenna (Ant-C), four radome mounted antennas (Ant-T, Ant$\mathrm{R}$, Ant-B, and Ant-L), and a supporting structure. The radome mounted antennas realize different main beam directions to the up, bottom, left, and right sides related to the platform axis direction, and the configurations of the antennas are determined to secure the center space of the platform where the Ant-C would be located, which can help to increase $\Sigma$-pattern gain of the monopulse antenna. The Ant-T\&B and Ant-L\&R are used for target DF on elevation (yz-plane) and azimuth (xz-plane) planes, respectively. Ant-C plays a role in enhancing $\Sigma$-pattern gain, which is related to the target detection range. All antennas have the same linear polarization on the $y$-axis to minimize the gain degradation from polarization mismatch. The radome profile is tangent ogive, and the material is $\mathrm{MC}$ Nylon, which has a

Manuscript received August 10, 2020 ; Revised October 29, 2020 ; Accepted December 8, 2020. (ID No. 20200810-118J)

Missile Research Institute, Agency for Defense Development, Daejeon, Korea.

"Corresponding Author: Jaesik Kim (e-mail: jaesikkim1028@gmail.com)

This is an Open-Access article distributed under the terms of the Creative Commons Attribution Non-Commercial License (http://creativecommons.org/licenses/by-nc/4.0) which permits unrestricted non-commercial use, distribution, and reproduction in any medium, provided the original work is properly cited.

(c) Copyright The Korean Institute of Electromagnetic Engineering and Science. 
permittivity of 3.05 and loss tangent of 0.0123 at center frequency $\left(=f_{c} \mathrm{GHz}\right)$. The inner radius and the thickness of the radome are $1.52 \lambda_{c}$ and $0.35 \lambda_{c}$, respectively.

Fig. 1(b) shows a cut-view of Ant-T in Fig. 1(a). Ant-B has the same configuration as Ant-T except for the length of the phase matching section. Ant-L\&R have similar composition to Ant-T\&B. To secure the center space of the radome where Ant-C is located, end-launch feeding is used, and the curved aperture is applied, which has the same curvature to the inner surface profile of the radome that makes the antenna structure as close to the radome as possible. These concepts can help to minimize the dimension of the antenna along the $y$-axis direction. The phase matching section performs to match the radiated phases of all antennas in the far-field region at the axis of the radome to obtain maximum gain in that direction. The stepped impedance matching structure performs not only impedance matching but also mode conversion from TEM to dominant modes between the connector and square open-ended waveguide. The curved aperture is used to extend an aperture to increase gain. In the case of Ant- $T$, the aperture is extended along the $\mathrm{xz}$-plane, and the curved aperture whose profile is the same as the inner radome surface shape is applied to secure the radome center space as much as possible. The reflector controls the main beam direction considering the radome effect.

For environmental robustness of the assembly from vibration

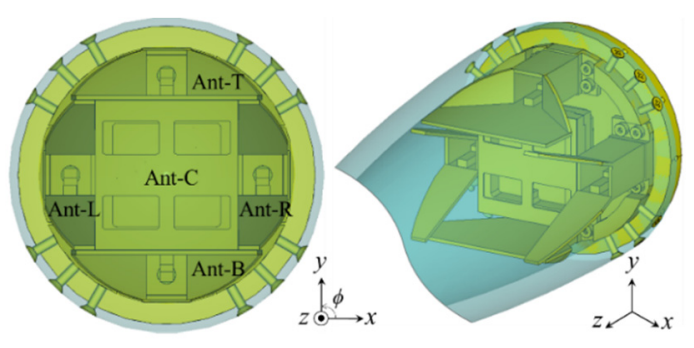

(a)

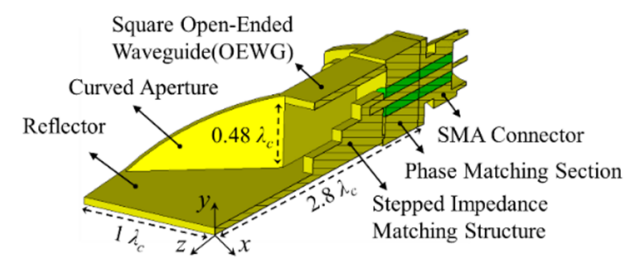

(b)

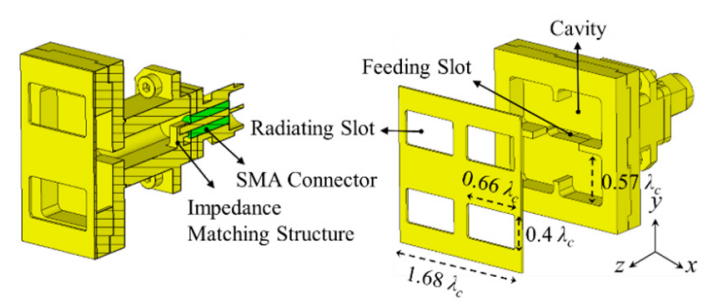

(c)

Fig. 1. Configurations of (a) proposed DF antenna assembly, (b) Ant-T, and (c) Ant-C. and heat environments, a metal antenna type is considered a candidate for Ant-C, such as horn, metal slot antenna, and so on. The antenna type should be selected to minimize the performance degradation of the radome mounted antennas when Ant-C is inserted at the center of them. In [6], a slot fed cavity antenna with compact dimension is presented which consists of a feeding waveguide, coupling slot, cavity, and radiation slot. The electric field in the waveguide is coupled to the feeding slot and the equal phase and amplitude are excited to the radiating slots due to its symmetric configurations of the cavity and radiating slots. Fig. 1(c) shows the configuration of the designed slot fed cavity antenna. Compared to the antenna presented in [6], the feeding method is modified from coupled slot feeding to end-launch feeding to minimize the antenna dimension on yz-plane. Along z-axis, designed Ant-C ends at the front end of the radiation part of the radome mounted antennas, which helps to minimize performance degradations of the radome mounted antennas.

\section{FABRICATION AND MEASUREMENT RESULTS}

Fig. 2 shows the fabricated antenna assembly. All antennas and supporting structures are made of aluminum. Fig. 3 shows the measured VSWR (voltage standing wave ratio) results of every antenna and far-field radiated phase differences at $f_{c} \mathrm{GHz}$. All results include radome effects. All antennas realize fractional bandwidth above $3.2 \%$ in $\mathrm{Ku}$-band $\left(f_{b} \mathrm{GHz}-f_{l} \mathrm{GHz}=3.2 \% \times\right.$ $f_{c} \mathrm{GHz}$ ). The maximum far-field radiated phase difference between all antennas is $12^{\circ}$ at the axis of the assembly. This error can be minimized by applying stable feeding systems, such as waveguide feeding with Magic-T structures, which can minimize the unstable phase errors from cables, power combiners, and so on. Fig. 4 illustrates the measured $\Sigma, \Delta$-patterns, and its $\Delta / \Sigma$ monopulse curves on the elevation and azimuth planes. To measure $\Sigma$-patterns, a five-way power combiner is used. Measured $\Delta$-patterns are the calculated results from the measured amplitudes and phases of the radiation patterns of every antenna. The reason that the null points of the $\Delta$-patterns are not perfectly positioned at $0^{\circ}$ is the radiated phase differences at the axis of the antenna assembly. The $\Sigma$-pattern gains are $16.80 \mathrm{dBi}$, $16.79 \mathrm{dBi}$, and $16.83 \mathrm{dBi}$ for $f_{l} \mathrm{GHz}, f_{c} \mathrm{GHz}$, and $f_{b} \mathrm{GHz}$, re-

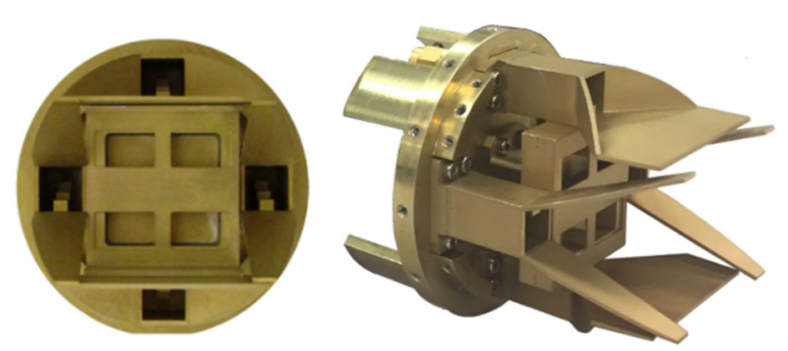

Fig. 2. Configuration of the proposed antenna assembly. 


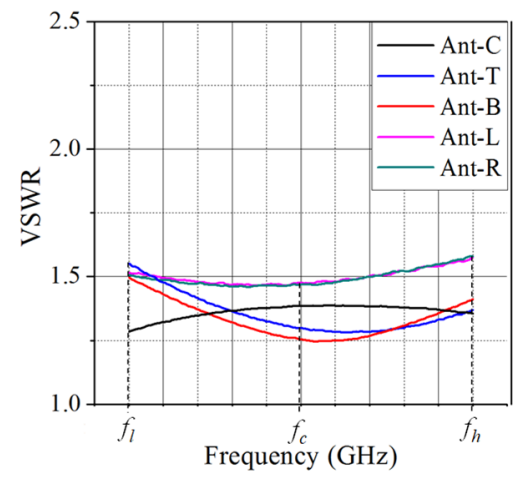

(a)

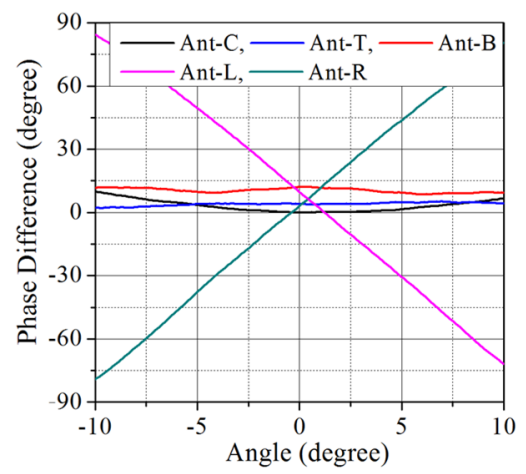

(b)

Fig. 3. Measurement results of (a) VSWR and (b) far-field radiated phase differences at $f_{c} \mathrm{GHz}$.
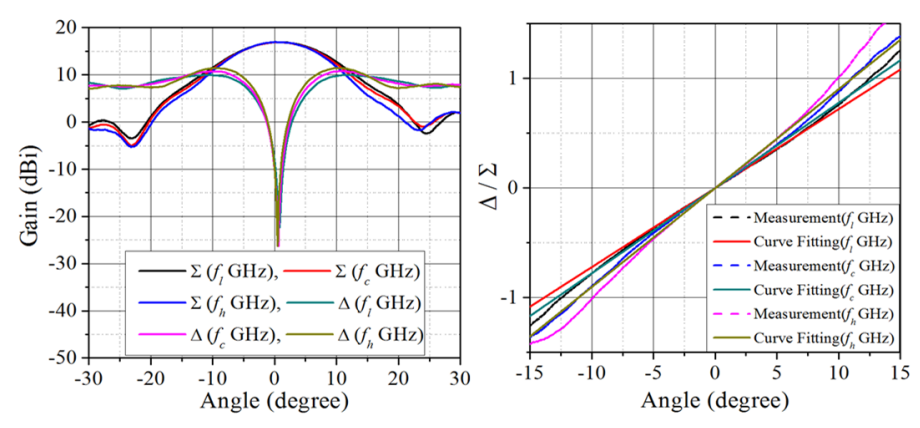

(a)
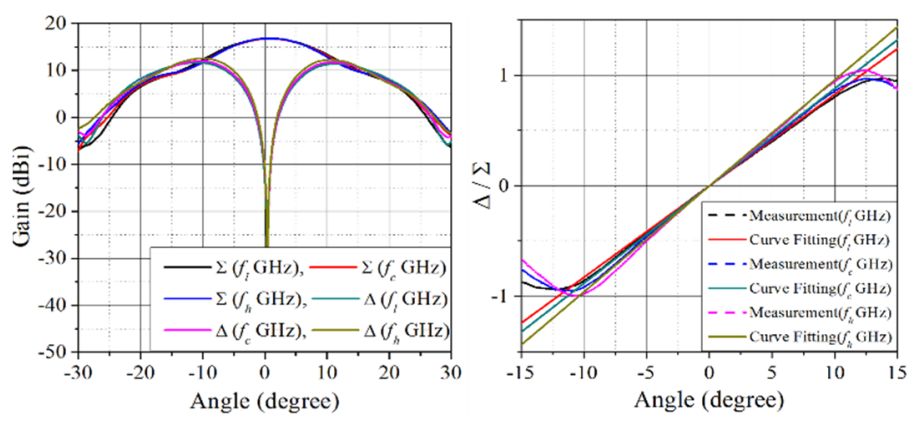

(b)

Fig. 4. Measured $\Sigma$-patterns and $\Delta / \Sigma$ monopulse curves on the (a) elevation and (b) azimuth planes.

spectively, which correspond to the antenna efficiency of above $52 \%$. The normalized null depths of the $\Delta$-patterns are under
$-40 \mathrm{~dB}$ which is a proper value considering boresight error of the antenna assembly. On the elevation plane, the monopulse slopes are $7.3 \%, 7.8 \%$, and $9.1 \%$ for $f_{l} \mathrm{GHz}, f_{c} \mathrm{GHz}$, and $f_{b} \mathrm{GHz}$, respectively, and its linear DF regions are above $\pm 5^{\circ}$. On the azimuth plane, the monopulse slopes are 8.2\%, 8.6\%, and 9.3\% for $f_{l} \mathrm{GHz}, f_{c} \mathrm{GHz}$, and $f_{b} \mathrm{GHz}$, respectively, and its linear DF regions are above $\pm 5^{\circ}$.

\section{CONCLUSION}

In this letter, a new configuration of a compact monopulse DF antenna assembly is presented which is composed of four radome mounted antennas and a slot fed cavity antenna. The radome mounted antenna is fed by end-launch feeding and has the curved aperture that profile is the same as the inner radome profile. These design concepts allow the antennas to locate as close to the inside surface of the radome as possible. As a result, the center space of the radome is secured and the space is used for placing the slot fed cavity antenna. In the operational bandwidth, $\Sigma$ pattern gains are $16.79-16.83 \mathrm{dBi}$ and the normalized null depths of the $\Delta$ patterns are under $-40 \mathrm{~dB}$, where the monopulse slopes are 7.3\%-9.3\%. From the results, it can be noticed that the presented assembly configuration can be a candidate for monopulse DF systems for small diameter flight vehicle applications.

\section{REFERENCES}

[1] S. M. Sherman and D. K. Barton, Monopulse Principle and Techniques, 2nd ed. Norwood, MA: Artech House, 2011.

[2] P. Zheng, G. Q.Zhao, S. H. Xu, F. Yang, and H.J. Sun, "Design of a W-band full-polarization monopulse Cassegrain antenna," IEEE Antennas and Wireless Propagation Letters, vol. 16, pp. 99-103, 2016.

[3] Y. W. Wang, G. M. Wang, Z. W. Yu, J. G. Liang, and X. J. Gao, "Ultra-wideband E-plane monopulse antenna using Vivaldi antenna," IEEE Transactions on Antennas and Propagation, vol. 62, no. 10, pp. 4961-4969, 2014.

[4] Y. J. Cheng, W. Hong, and K. Wu, "Design of a monopulse antenna using a dual $\mathrm{V}$-type linearly tapered slot antenna (DVLTSA)," IEEE Transactions on Antennas and Propagation, vol. 56, no. 9, pp. 2903-2909, 2008.

[5] A. K. Keskin, M. A. Tulum, and A. S. Turk, "Quasi horn antenna array for Ku band monopulse radiation," in Proceedings of 2016 17th International Radar Symposium (IRS), Krakow, Poland, 2016, pp. 1-3.

[6] Y. Miura, J. Hirokawa, M. Ando, Y. Shibuya, and G. Yoshida, "Double-layer full-corporate-feed hollow-waveguide slot array antenna in the $60-\mathrm{GHz}$ band," IEEE Transactions on Antennas and Propagation, vol. 59, no. 8, pp. 2844-2851, 2011. 